Check for updates

Cite this: Chem. Sci., 2020, 11, 62

๑ All publication charges for this article have been paid for by the Royal Society of Chemistry

Received 14th July 2019

Accepted 15th October 2019

DOI: $10.1039 / c 9 s c 03469 b$

rsc.li/chemical-science

\section{RNA imaging in living mice enabled by an in vivo hybridization chain reaction circuit with a tripartite DNA probe $\uparrow$}

\author{
Han Wu, ${ }^{\text {ab }}$ Ting-Ting Chen, (D) ${ }^{a}$ Xiang-Nan Wang, ${ }^{a}$ Yonggang Ke (D) *b \\ and Jian-Hui Jiang (iD) *a
}

RNA imaging in living animals helps decipher biology and creates new theranostics for disease treatment. Due to their low delivery efficiency and high background, however, fluorescence probes for in situ RNA imaging in living mice have not been reported. We develop a new cell-targeting fluorescent probe that enables RNA imaging in living mice via an in vivo hybridization chain reaction (HCR). The minimalistic $Y$ shaped design of the tripartite DNA probe improves its performance in live animal studies and serves as a modular scaffold for three DNA motifs for cell-targeting and the HCR circuit. The tripartite DNA probe allows facile synthesis with a high yield and demonstrates ultrasensitive RNA detection in vitro. The probe also exhibits selective and efficient internalization into folate (FA) receptor-overexpressed cells via a caveolar-mediated endocytosis mechanism and produces fluorescence signals dynamically correlated with intracellular target expressions. Furthermore, the probe exhibits specific delivery into tumor cells and allows high-contrast imaging of miR-21 in living mice. The tripartite DNA design may open the door for intracellular RNA imaging in living animals using DNA-minimal structures and its design strategy can help future development of DNA-based multi-functional molecular probes.

\section{Introduction}

Molecular probes that enable imaging of specific molecular and cellular processes in living animals are indispensable tools in deciphering pathological biology and theranostics for different diseases including cancer. ${ }^{\mathbf{1 , 2}}$ Current in vivo imaging probes for cancer are mostly developed by targeting tumor-associated enzymes, ${ }^{3-5}$ protein receptors, ${ }^{6,7}$ and microenvironments, ${ }^{8-10}$ which only comprise a considerably small subset of the total hallmarks of cancer. ${ }^{11}$ Given the crucial biological roles and the increasing number of RNA-based biomarkers identified, ${ }^{\mathbf{1 2}}$ fluorescent probes targeting RNAs have become a valuable platform to gain insight into tumor biology and theranostic cancer treatment.

Fluorescent probes for RNA imaging can be engineered with two different strategies. One utilizes fluorescent protein-tagged RNA-binding proteins for labeling and tracking of RNAs, ${ }^{13}$ and the other deploys hybridization probes such as molecular

${ }^{a}$ State Key Laboratory of Chemo/Bio-Sensing and Chemometrics, College of Chemistry and Chemical Engineering, Hunan University, Changsha 410082, P. R. China. E-mail: jianhuijiang@hnu.edu.cn

${ }^{b}$ Wallace H. Coulter Department of Biomedical Engineering, Emory University School of Medicine, Emory University, Atlanta, Georgia 30322, USA. E-mail: yonggang.ke@ emory.edu

$\dagger$ Electronic supplementary information (ESI) available. See DOI: $10.1039 / \mathrm{c} 9 \mathrm{sc} 03469 \mathrm{~b}$ beacons, ${ }^{\mathbf{1 4 , 1 5}}$ spherical nucleic acids ${ }^{\mathbf{1 6 , 1 7}}$ and light-up RNA aptamers $^{18,19}$ for activatable detection of RNAs. However, current probes have only been used for imaging in living cells $^{13-19}$ or sliced tissues from living animals..$^{20-22}$ The development of fluorescent probes for imaging of endogenous RNA in living animals remains highly elusive. ${ }^{23} \mathrm{~A}$ key challenge for RNA imaging probes is their delivery into specific cells due to their nuclease-mediated degradation within body fluids and low efficiency in cellular uptake, which is also the substantial obstacle to oligonucleotide therapeutics. ${ }^{24}$ Another major hurdle is the low abundance of RNA targets, which only generate a weak fluorescence response in the absence of signal amplification. To our knowledge, although hybridization chain reaction probes for fluorescence in situ hybridization (FISH) in various model organisms such as zebrafish have been used in recent years, ${ }^{25,26}$ fluorescent probes that have the ability to achieve in vivo RNA imaging in living mammalian animals have been unexplored. Here we develop a novel fluorescent probe that for the first time enables RNA imaging in living mice via an in vivo hybridization chain reaction (HCR) ${ }^{27}$ using a celltargeting tripartite DNA probe. Our previous study had established that a HCR based DNA amplification circuit allowed highly sensitive imaging of mRNA in living cells. ${ }^{28}$ The advantage of using DNA amplification circuits for RNA imaging is the enzyme-free signal amplification with hundreds of fluorophores activated from a single RNA. We envision that the realization of 
an in vivo HCR circuit affords the possibility of visualizing lowabundance RNAs in living animals.

Motivated by this hypothesis, we design a probe using a tripartite Y-shaped DNA for in vivo HCR circuit-based RNA imaging, as illustrated in Scheme 1. The use of a Y-shaped DNA structure has two advantages. One is the tripartite modular scaffold conferring three modules for functionalization with three DNA motifs required for an in vivo HCR circuit. The other is that the Y-shape is a DNA-minimal structure with tripartite functionalities.

The minimal complexity of the probe can enhance its performance in live animal studies compared to alternative DNA structures with more strands. Three DNA motifs are assembled on the Y-shaped probe via sticky-end hybridization (Scheme 1A). One motif is a cell-targeting DNA-peptide (polylysine) conjugated folate probe (FAP), in which the poly-lysine domain is grafted with multiple folate (FA) ligands. Since tumor cells are mostly known for overexpression of folate receptors ${ }^{29}$ such a multiple ligand presentation in the DNA probe can confer improved affinity and targetability to tumor cells through multivalent interactions. The other two motifs are hairpin probes $\mathrm{H} 1$ and $\mathrm{H} 2$, which undergo HCR assembly in

A
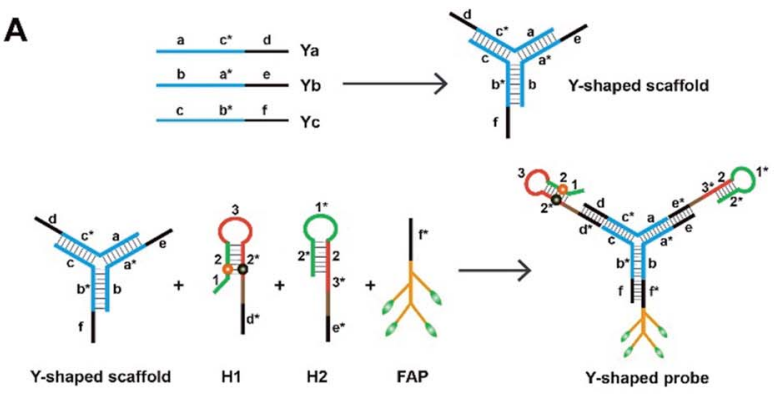

B
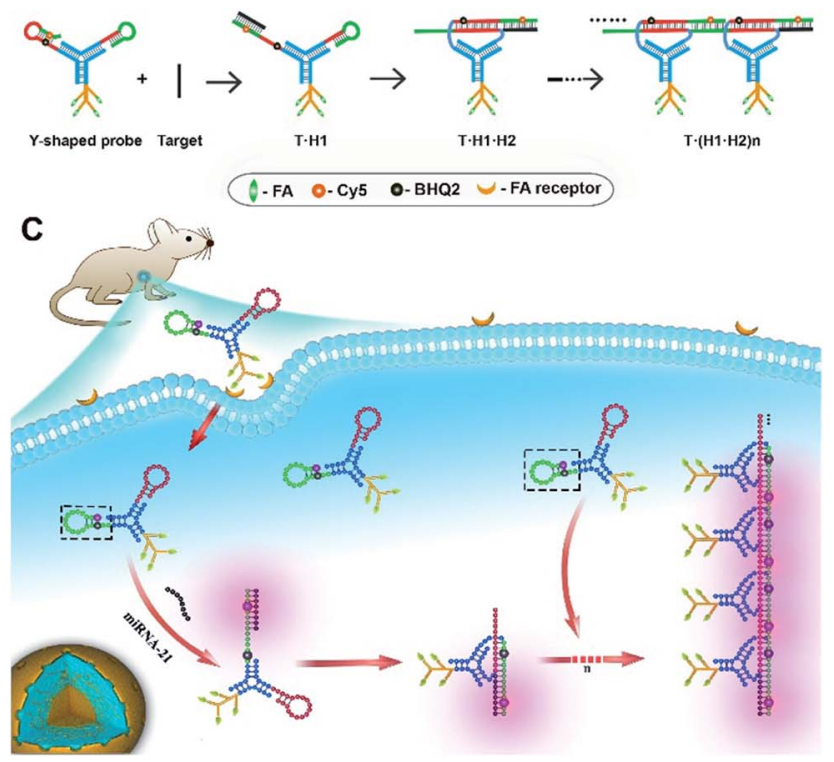

Scheme 1 Illustration of design of the tripartite $Y$-shaped DNA probe (A), a detailed illustration of the alternating assembly (B) and in vivo HCR circuit for RNA imaging via intravenous injection (C). response to a target RNA (Scheme 1B). First, the target miR-21 hybridizes with the toehold domain 1 and domain 2 of $\mathrm{H} 1$ and opens the hairpin. Then the activated domain 3 and domain $2 *$ in $\mathrm{H} 1$ hybridize with the domain $3^{*}$ and domain 2 of $\mathrm{H} 2$ and expose the domain $1^{*}$ and $2^{*}$ for the next cycle of the HCR reaction. A near-infrared (NIR) fluorescence reporter (Cy5) and a quencher moiety BHQ2 are labeled in the stem region of the $\mathrm{H} 1$ probe such that a fluorescence signal is activated to indicate the HCR assembly. When systemically administered into a living mouse via intravenous injection, the tripartite DNA probe can be selectively taken up into tumor cells. The presence of target RNA mediates a cascade of alternating assembly between probes $\mathrm{H} 1$ and $\mathrm{H} 2$ via the HCR circuit, generating an activated NIR fluorescence response for in vivo RNA imaging in the living mouse (Scheme 1C).

As a proof of principle, we choose microRNA-21 (miR-21) as the target in this work, because of its overexpression in various tumor cells. ${ }^{30}$ We demonstrate that the tripartite DNA probe allows facile modular synthesis with a high yield and ultrasensitive detection of target RNA in vitro. Live cell assays reveal that the tripartite DNA allows selectively and efficiently internalization in cells with FA receptor overexpression, displaying fluorescence signals dynamically correlated with miR-21 expression. Additionally, its caveolar-mediated endocytosis mechanism affords the potential for efficient escape from lysosomal degradation. Moreover, the tripartite DNA probe affords increased stability of the probe in serum and enables specific delivery into tumor cells for high-contrast imaging of miR-21 in living mice. Because of the broad utility of DNA amplification circuits for RNA detection, the tripartite DNA design may open the door for intracellular RNA imaging in living animals using DNA-minimal structures.

\section{Results and discussion}

\section{Design and synthesis of the tripartite DNA probe}

Three motifs are required to realize the in vivo HCR circuit: a cell-targeting unit to deliver the hairpin probes into tumor cells and two hairpin probes responding to target RNA to initiate the HCR assembly. We chose a DNA-minimal Y-shaped scaffold $^{31,32}$ to construct the modular probe for in vivo realization of the HCR circuit. Such a DNA-minimal structure afforded the advantages of low cost and high efficiency in synthesis and minimized complexity of structural variability for in vivo applications. The Y-shaped DNA was synthesized via self-assembly using three 39-mer single-stranded (ss) DNAs ( $\mathrm{Ya}, \mathrm{Yb}$ and $\mathrm{Yc}$ ), each containing a 26-mer region to form the double-stranded $\mathrm{Y}$ shape and a 13-mer sticky end of different sequences. To synthesize a cell-targeting motif, a poly-lysine peptide with an azido modifier, azido-GGKKKKKKKK, was designed, in which the side chain amino groups were conjugated to multiple FA using the EDC-NHS chemistry (Fig. S1 $\dagger$ ). The cell-targeting motif, DNA-peptide conjugated FAP, was synthesized by linking the FA conjugated peptide to a ssDNA linker L complementary to a sticky end with a $5^{\prime}$-alkynyl modifier via copper catalyzed click chemistry ${ }^{33}$ (Fig. S2 and S3†). The hairpin probes $\mathrm{H} 1$ and $\mathrm{H} 2$ were carefully designed and optimized according to 
the miR-21 sequence to minimize the background arising from reaction with non-homologous initiator sequences (Fig. S4 $\dagger$ ). With the cell-targeting DNA-peptide conjugated FAP and hairpin probes $\mathrm{H} 1$ and $\mathrm{H} 2$, the tripartite DNA probe $\mathrm{Y}-\mathrm{H} 1-\mathrm{H} 2-$ FAP was synthesized via assembling the three motifs on the corresponding sticky ends.

Gel electrophoresis analysis was used to validate the successful synthesis of the tripartite DNA probe (Fig. 1A). A major band with lower mobility appeared after incubation of the three component ssDNAs ( $\mathrm{Ya}, \mathrm{Yb}$ and $\mathrm{Yc}$ ), indicating the formation of a Y-shaped scaffold from the three component ssDNAs. With addition of one of the three motifs, major bands were observed with further lowered mobility, verifying the high efficiency in assembly of each of the three motifs on the Yshaped scaffold. A one-pot synthesis using three component ssDNAs (Ya, Yb and Yc) and three motifs (FAP, H1 and H2) also only yield a major band with substantially lower mobility, evidencing the formation of the complete tripartite DNA probe. The estimated yield of the Y-shaped scaffold was close to $100 \%$ and that of the tripartite DNA probe was $\sim 90 \%$, as determined by densitometry. The high yield, facile one-pot synthesis and minimal design of the tripartite DNA probe afforded unique

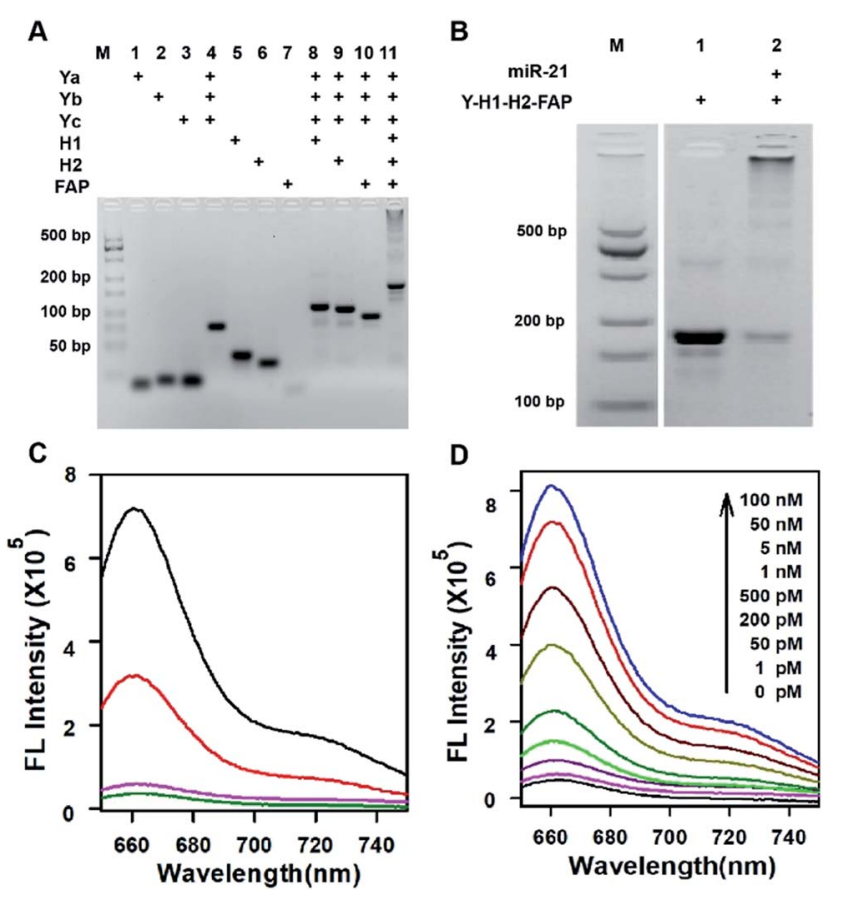

Fig. 1 (A) Agarose gel (4\%) electrophoresis image of the tripartite DNA probe $\mathrm{Y}-\mathrm{H} 1-\mathrm{H} 2-\mathrm{FAP} . \mathrm{M}$ denotes molecular weight marker. The concentration of each DNA probe in the well is $1 \mu \mathrm{M}$. (B) Agarose gel (4\%) electrophoresis image of HCR assay. $M$ denotes molecular weight marker. The concentrations of the probe $\mathrm{Y}-\mathrm{H} 1-\mathrm{H} 2-\mathrm{FAP}$ and target miR-21 are $1 \mu \mathrm{M}$ and $500 \mathrm{nM}$ respectively. (C) Fluorescence spectra of the DNA probe $\mathrm{Y}-\mathrm{H} 1-\mathrm{H} 2-\mathrm{FAP}$ (black) and $\mathrm{Y}-\mathrm{H} 1-\mathrm{FAP}$ (red) incubated with miR-21, and $\mathrm{Y}-\mathrm{H} 1-\mathrm{H} 2-\mathrm{FAP}$ without the target (green) or with a control RNA (pink). The concentrations of the probe $\mathrm{Y}-\mathrm{H} 1-\mathrm{H} 2-\mathrm{FAP}$ and $\mathrm{Y}-\mathrm{H} 1-\mathrm{FAP}$ are $100 \mathrm{nM}$, and the concentrations of the target miR21 and control RNA are $50 \mathrm{nM}$. (D) Fluorescence spectral responses of the DNA probe $\mathrm{Y}-\mathrm{H} 1-\mathrm{H} 2-\mathrm{FAP}$ to $\mathrm{miR}-21$ of varying concentrations. The concentration of the probe $\mathrm{Y}-\mathrm{H} 1-\mathrm{H} 2-\mathrm{FAP}$ is $100 \mathrm{nM}$. advantages for its living animal applications that typically require using probes at a relatively high dosage.

\section{In vitro evaluation of the tripartite DNA probe for RNA detection}

Because of the minimal structure of the tripartite DNA probe with minimized steric hindrance, we expected that it still allowed an efficient HCR assembly in response to target miR-21. Gel electrophoresis analysis demonstrated that upon incubation with the target miR-21, the tripartite DNA probe $\mathrm{Y}-\mathrm{H} 1-\mathrm{H} 2-$ FAP gave consecutive bands with decreased mobility, consistent with reported HCR circuits (Fig. 1B). The majority of HCR products were trapped in the well, indicating efficient assembly of the tripartite DNA probes into large HCR polymers. This finding verified the feasibility of using the tripartite DNA probe to realize the HCR circuit in response to miR-21. Further fluorescence assays revealed that the tripartite DNA probe $\mathrm{Y}-\mathrm{H} 1-$ H2-FAP gave substantial enhancement ( 15 -fold) in the Cy5 fluorescence signal after incubating with $50 \mathrm{nM}$ target RNA (Fig. 1C). In contrast, a control RNA with the most homogeneity to miR-21 in mammalian cells only generated a very slight increase in the fluorescence peak, suggesting the high specificity of the HCR circuit using the tripartite DNA probe. A control Y-shaped probe Y-H1-FAP, which can also be activated by miR-21 but with no further signal amplification, showed only $40 \%$ fluorescence output upon interaction with $50 \mathrm{nM}$ miR-21, which can directly trigger maximally the opening of half of the probe $\mathrm{Y}-\mathrm{H} 1-\mathrm{FAP}$. These results gave direct evidence for selective and signal-amplified fluorescence activation of the tripartite DNA probe in response to target miR-21. Further investigation revealed that the tripartite DNA probe exhibited dynamic responses to miR-21 of varying concentrations from $1 \mathrm{pM}$ to $100 \mathrm{nM}$ (Fig. 1D). The detection limit was estimated to be 0.8 pM according to the linear correlation between peak intensities and target concentrations (Fig. S5 $\dagger$ ). Moreover, the tripartite DNA probe displayed fast kinetics in the HCR assembly and the reactions almost completed within 40 min (Fig. S6†).

\section{Detection of miR-21 in live cells with the tripartite DNA probe}

To test the performance of the tripartite DNA probe for imaging of miR-21 in living cells, we chose a model system of HeLa cells, which was known for overexpression of the target miR-21 and FA receptor on the cell membrane. ${ }^{34,35}$ After HeLa cells were incubated with the tripartite DNA probe $\mathrm{Y}-\mathrm{H} 1-\mathrm{H} 2-\mathrm{FAP}$ (100 $\mathrm{nM}$ ) for $3 \mathrm{~h}$, we observed bright red fluorescence in the cells (Fig. 2A, image 1). Confocal images at higher resolution showed highly localized fluorescent spots around the nuclei (Fig. S7†), implying that the tripartite DNA probe based HCR circuit had potential for localization detection of target RNA. Timedependent imaging further revealed that the cells started to show fluorescence after $20 \mathrm{~min}$ incubation and the signal became saturated after $60 \mathrm{~min}$ (Fig. S8 $\dagger$ ). This observation suggested that the in cellular HCR kinetics was close to that under in vitro conditions. A control experiment using a $\mathrm{Y}$ shaped probe $\mathrm{Y}-\mathrm{H} 1-\mathrm{H} 2$ gave no detectable fluorescence in the cells (Fig. 2A, image 2), indicating that the cell-targeting motif 
A
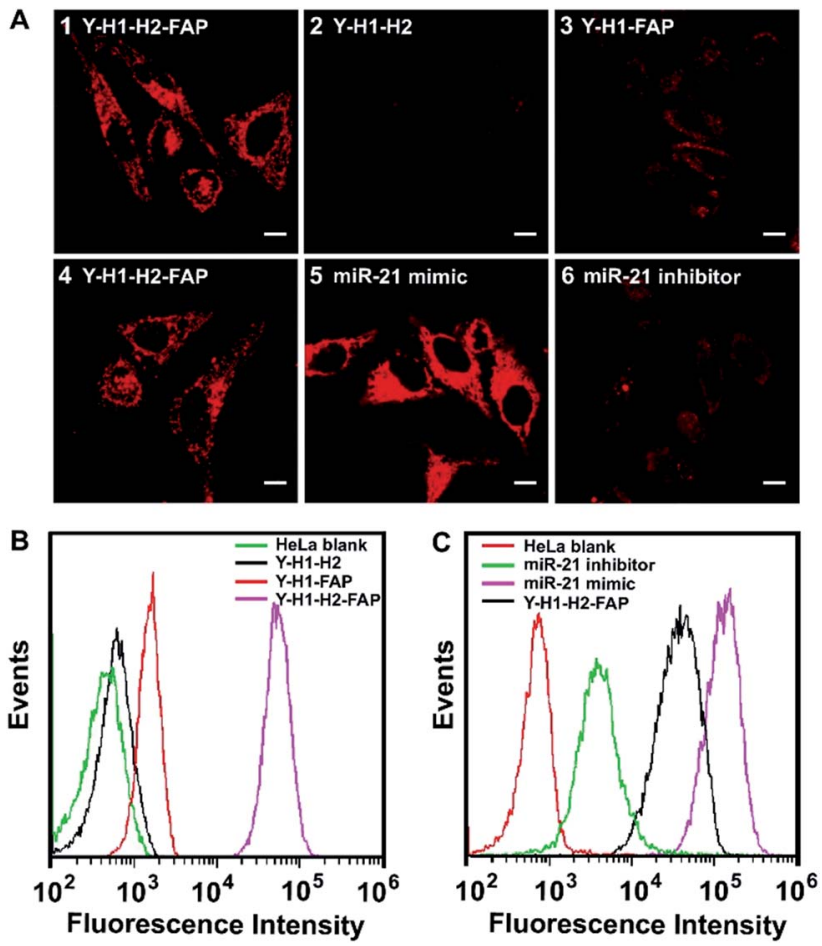

Fig. 2 (A) Fluorescence images of HeLa cells treated with the $\mathrm{Y}-\mathrm{H} 1-$ $\mathrm{H} 2-\mathrm{FAP}$ probe (1 and 4), $\mathrm{Y}-\mathrm{H} 1-\mathrm{H} 2$ probe (2) and $\mathrm{Y}-\mathrm{H} 1-\mathrm{FAP}$ probe (3). HeLa cells pretreated with a $300 \mathrm{nM}$ miR-21 mimic (5) and $300 \mathrm{nM}$ inhibitor (6) followed by incubation with the $\mathrm{Y}-\mathrm{H} 1-\mathrm{H} 2-\mathrm{FAP}$ probe. The concentrations of the probe $\mathrm{Y}-\mathrm{H} 1-\mathrm{H} 2-\mathrm{FAP}, \mathrm{Y}-\mathrm{H} 1-\mathrm{H} 2$ and $\mathrm{Y}-$ $\mathrm{H} 1-\mathrm{FAP}$ are $100 \mathrm{nM}$. (B and C) Flow cytometry profiles of the corresponding HeLa cells. The HeLa blank represents HeLa cells without any treatment.

FAP played an essential role in cellular delivery of the DNA probe. An additional control experiment using the nonamplification Y-shaped probe $\mathrm{Y}-\mathrm{H} 1-\mathrm{FAP}$ only exhibited weak fluorescence in the cells (Fig. 2A, image 3), suggesting that the tripartite DNA probe based HCR circuit afforded substantial sensitivity enhancement in live cell imaging of miR-21. Flow cytometry assay gave remarkably increased fluorescence signals for the tripartite DNA probe $\mathrm{Y}-\mathrm{H} 1-\mathrm{H} 2-\mathrm{FAP}$ over the nonamplification probe $\mathrm{Y}-\mathrm{H} 1-\mathrm{FAP}$ and further over the nontargeting probe $\mathrm{Y}-\mathrm{H} 1-\mathrm{H} 2$ (Fig. 2B). The results above demonstrated that the tripartite DNA probe $\mathrm{Y}-\mathrm{H} 1-\mathrm{H} 2-\mathrm{FAP}$ allowed efficient cell uptake and substantial signal amplification.

The specific response of the tripartite DNA probe to miR-21 was validated using HeLa cells treated with miR-21 mimic, a synthetic dsRNA enabling the increase of miR-21 expression, ${ }^{36}$ or miR-21 inhibitor, a synthetic ssRNA complementary to miR21 and decreasing its expression. ${ }^{36}$ Confocal images revealed that cells pretreated with the miR-21 mimic displayed stronger fluorescence than untreated HeLa cells, while cells pretreated with the miR-21 inhibitor gave much lower fluorescence (Fig. 2A, image 4-6). Flow cytometry analysis also showed consistent fluorescence responses in a decreasing order of HeLa cells treated with the miR-21 mimic, untreated cells and cells treated with the miR-21 inhibitor (Fig. 2C). These findings revealed a strong correlation between the miR-21 concentration and the fluorescence signals generated by the tripartite DNA probe, indicating the probe's potential for quantitative detection of target RNA in living cells.

\section{FA receptor-specific endocytosis of the tripartite DNA probe}

To realize in vivo RNA imaging in tumors, it is critical for the tripartite DNA probe to enable cell-targeting delivery into tumor cells. Because a culture medium containing FA could block the receptors, ${ }^{37}$ we started to investigate the targeting properties of the tripartite DNA probe to FA receptor-overexpressed tumor cells using HeLa cells incubated in a culture medium with or without FA. As anticipated, after growing in the culture medium containing FA, HeLa cells incubated with the tripartite DNA probe did not show appreciable fluorescence contrast (Fig. S9 $\dagger$ ), which was also consistent with the data obtained with flow cytometry. This finding suggested that the tripartite DNA probe entered the cells via a FA receptor dependent mechanism. Further evidence was obtained with MCF-7 cells, which were known to have negative expression of the FA receptor but overexpression of the miR-21 target. ${ }^{38,39}$ After incubation with the tripartite DNA probe, MCF-7 cells displayed a negligible fluorescence signal (Fig. S9†), confirming the FA receptorspecific internalization of the tripartite DNA probe in tumor cells.

To further validate the FA receptor-specific cellular uptake of the tripartite DNA probe, a plasmid that induces overexpression of the FA receptor (Scheme $\mathrm{S} 1 \dagger$ ) was used for transfection of two cell lines, L-02 and MCF-7, both with low expression of the FA receptor. ${ }^{39}$ After the transfected cells were incubated with the tripartite DNA probe, we observed fluorescence images as well as flow cytometry profiles of varying fluorescence intensities in these cells (Fig. S10 $\dagger$ ). MCF-7 cells displayed the brightest fluorescence, indicating the highest expression of miR-21. L-02 cells showed the weakest fluorescence with an indicator for the lowest expression of miR-21. The results were consistent with previous reports $^{38}$ and our RT-PCR analysis (Fig. S11†). These findings confirmed the FA receptor-specific cellular uptake of the tripartite DNA probe and served as additional evidence for quantitative imaging of miR-21 in living cells with the tripartite DNA probe.

Furthermore, the intracellular uptake mechanism was investigated using inhibitors for different cellular internalization pathways ${ }^{\mathbf{4 0}}$ with a fluorescent tripartite DNA probe $\mathrm{Y}-\mathrm{H} 3-$ H4-FAP (Fig. S12 $\dagger$ ). The tripartite DNA probe Y-H3-H4-FAP replaced $\mathrm{H} 1$ with a non-responsive hairpin probe $\mathrm{H} 3$ and a TARMA labeled version $\mathrm{H} 4$ for the hairpin probe $\mathrm{H} 2$. When HeLa cells were incubated with the fluorescent tripartite DNA probe in the presence of $\mathrm{NaN}_{3}$, no obvious fluorescence appeared in the cells. As $\mathrm{NaN}_{3}$ was an inhibitor for ATPase involved in all energy-dependent endocytosis, ${ }^{40}$ this finding confirmed an energy-dependent endocytosis pathway for the tripartite DNA probe. It was also found that cells treated with nystatin (caveolar mediated endocytosis inhibitor ${ }^{\mathbf{4 0}}$ ) also displayed weak fluorescence after incubation with the fluorescent tripartite DNA probe. In contrast, chlorpromazine (CPZ, 
clathrin-mediated endocytosis inhibitor ${ }^{40}$ ), amiloride (macropinocytosis mediated endocytosis inhibitor ${ }^{40}$ ) and methyl $\beta$ cyclodextrin (lipid-raft mediated endocytosis inhibitor ${ }^{40}$ ) had a slight effect on the intracellular fluorescence signals. These results revealed that the tripartite DNA probe entered HeLa cells dominantly via a caveolar-mediated endocytosis mechanism. This finding was also consistent with previous observations that multivalent FA conjugated probes internalized into FA receptorpositive cells via caveolar-mediated endocytosis. ${ }^{41}$

A further time-dependent study revealed that the tripartite DNA probe Y-H3-H4-FAP did not display substantial colocalization with Lysotracker during the endocytosis (Fig. S13†). This observation was consistent with its caveolar-mediated endocytosis pathway in which caveosomes did not fuse with lysosomes. ${ }^{41}$ Because this caveolar mediated endocytosis mechanism allowed efficient escape from lysosomal degradation, we expected that the tripartite DNA probe could hold great promise for the delivery of nucleic acid agents for theranostics. Further evidence for the cytoplasm localization of the tripartite DNA probe $\mathrm{Y}-\mathrm{H} 1-\mathrm{H} 2-\mathrm{FAP}$ was obtained using colocalization assay of the HCR products with Lysotracker (Fig. S14†). It was also observed that the HCR products had little colocalization with lysosomes, implying that the tripartite DNA probe entered the cytoplasm and formed a HCR assembly in response to target miR-21. Additionally, a $z$-axis scan experiment further clearly demonstrated that the HCR products were localized in the cytoplasm (Fig. S15†).

Additionally, the tripartite DNA probe was found to show better stability in $100 \%$ fresh fetal bovine serum (FBS) than free hairpin probes $\mathrm{H} 1$ and $\mathrm{H} 2$ in a fluorescence assay (Fig. S16†). This finding was consistent with previous studies on the stability of other DNA probes. ${ }^{42,43}$ This result suggested that the tripartite DNA probe improved nuclease resistance of the hairpin probes, implying the potential of the tripartite DNA probe for in vivo imaging applications.

\section{RNA imaging in living mice with the tripartite DNA probe}

After validating the in vitro and in cellular performance, we then investigated the ability of the tripartite DNA probe for RNA imaging in living mice. We generated tumor xenografts via subcutaneous implantation of MCF-7 cells or HeLa cells $\left(>10^{6}\right.$ cells) into the right oxter of BALB/c mice. Imaging experiments were performed after the tumors were grown to a $20-25 \mathrm{~mm}^{3}$ size. In the preliminary study, we inspected the feasibility of the tripartite DNA probe for in vivo fluorescence imaging of miR-21 expression in tumors via intratumoral injection. With the injection of the physiological saline, very low background fluorescence was obtained (Fig. 3A, image 1). The intratumoral injection of the tripartite DNA probe in MCF-7 tumor bearing mice exhibited a weak fluorescence signal (Fig. 3A, image 2), attributed to the poor efficiency of the tripartite DNA probe to enter the tumor cells because of the negative expression of the FA receptor on their membranes. An intense fluorescence signal was observed for the HeLa tumor bearing mice (Fig. 3A, image 3), suggesting high expression of miR-21 in HeLa tumors with positive expression of the FA receptor on their membranes.
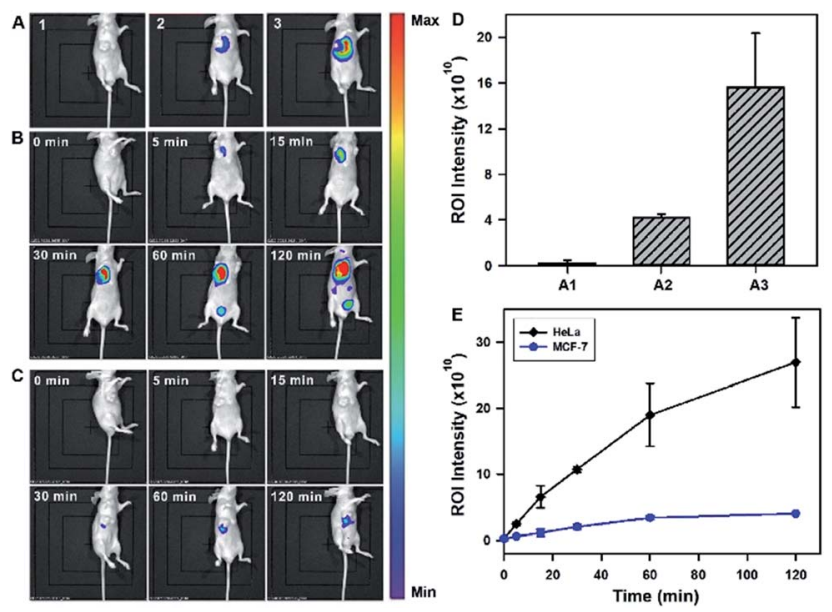

Fig. 3 (A) In vivo fluorescence images obtained $1 \mathrm{~h}$ after intratumoral injection for HeLa tumor-bearing mice with physiological saline injection (1), MCF-7 tumor-bearing mice with injection of the $\mathrm{Y}-\mathrm{H} 1-$ $\mathrm{H} 2-\mathrm{FAP}$ probe (2), and HeLa tumor-bearing mice with injection of the $\mathrm{Y}-\mathrm{H} 1-\mathrm{H} 2-\mathrm{FAP}$ probe (3). Time-dependent in vivo fluorescence images of HeLa (B) and MCF-7 (C) tumor-bearing mice with intratumoral injection of the $\mathrm{Y}-\mathrm{H} 1-\mathrm{H} 2-\mathrm{FAP}$ probe. (D) Mean fluorescence intensities of the treated mice in (A1)-(A3). (E) Profiles of the timedependent in vivo fluorescence intensities of tumor-bearing mice with injection of the $\mathrm{Y}-\mathrm{H} 1-\mathrm{H} 2-\mathrm{FAP}$ probe.

Clearer evidence for the overexpression of miR-21 in the HeLa tumor could be obtained from fluorescence intensities in the tumor regions of interest (Fig. 3D). Time-dependent in vivo imaging also exhibited a rapid increase in the fluorescence responses in the HeLa tumors at $2 \mathrm{~h}$ post-injection (Fig. 3B), and only slightly increased fluorescence signals were observed for the MCF-7 tumors (Fig. 3C). The ratio of average fluorescence signals for the HeLa tumors to the MCF-7 tumors at $2 \mathrm{~h}$ postinjection was as high as $\sim 7$-fold (Fig. 3E), indicating the high contrast of the tripartite DNA probe for miR-21 imaging in living mice.

With the desirable results obtained through intratumoral injection, we started to investigate the capability of the tripartite DNA probe for miR-21 imaging in living mice through tail vein administration. $1 \mathrm{~h}$ after the injection of the tripartite DNA probe, mice with no implanted tumor (Fig. 4A, image 1) or with MCF-7 tumors (Fig. 4A, image 2) only displayed very weak fluorescence signals, while the HeLa tumor exhibited strong fluorescence (Fig. 4A, image 3). The average intensities in the region of interest showed that HeLa tumor delivered $\sim 4$-fold enhancement in the fluorescence signals compared to MCF-7 tumors (Fig. 4C). This result evidenced that the tripartite DNA probe enabled rapid cell-targeted delivery of the probe and allowed specific fluorescence activation in response to the miR21 target. Further experiments using HeLa tumor bearing mice by tail vein administration of physiological saline (Fig. 4A, image 4), the non-amplification control probe Y-H1-FAP (Fig. 4A, image 5) and the non-targeting probe $\mathrm{Y}-\mathrm{H} 1-\mathrm{H} 2$ (Fig. 4A, image 6) also did not give an intense fluorescence signal. These controls confirmed that the FAP moiety was essential for cell-targeted delivery of the probe and signal 
A
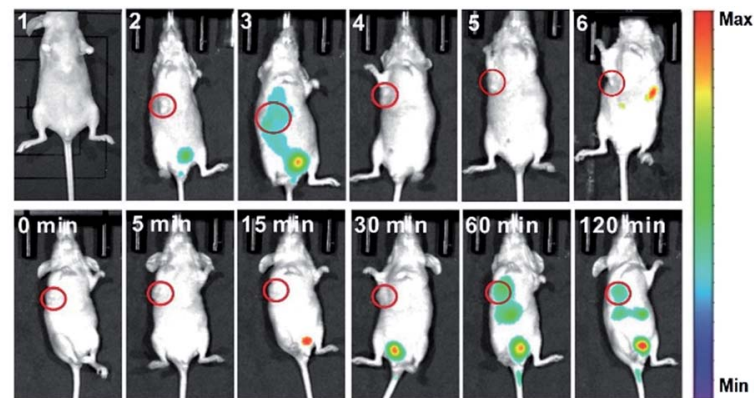

C

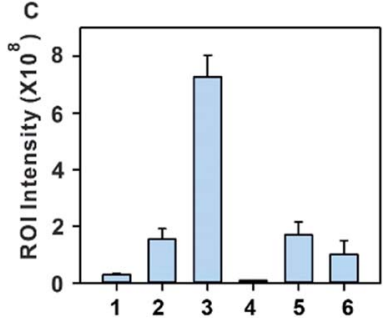

Fig. 4 (A) In vivo fluorescence images of mice obtained $1 \mathrm{~h}$ after intravenous injection. Mice with no implanted tumor (1), MCF-7 tumor (2) and HeLa tumor (3) were intravenously injected with the $\mathrm{Y}-\mathrm{H} 1-$ $\mathrm{H} 2-\mathrm{FAP}$ probe. HeLa tumor-bearing mice injected with physiological saline (4), the $\mathrm{Y}-\mathrm{H} 1-\mathrm{FAP}$ probe (5) and the $\mathrm{Y}-\mathrm{H} 1-\mathrm{H} 2$ probe (6). (B) Time-dependent in vivo fluorescence imaging of HeLa tumor-bearing mice intravenously injected with the $\mathrm{Y}-\mathrm{H} 1-\mathrm{H} 2-\mathrm{FAP}$ probe. (C) Mean fluorescence intensities of the treated mice in (A1)-(A6). (D) Profiles of the time-dependent in vivo fluorescence intensities of tumor-bearing mice with injection of a specified probe.

amplification using the HCR circuit was necessary for achieving high contrast imaging of RNA in living animals. Timedependent imaging of the HeLa tumor bearing mice showed that the fluorescence signals increased gradually after tail vein administration of the tripartite DNA probe and reached the maximum at $1 \mathrm{~h}$ followed by a decrease of the response (Fig. 4B). Besides the tumor region, fluorescence signals were also obtained in the bladder region, indicating a rapid clearance of the tripartite DNA probe through the kidney. In contrast, a control experiment of time-dependent imaging of the HeLa tumor bearing mice by injection of the control probe $\mathrm{Y}-\mathrm{H} 1-\mathrm{H} 2$ also only displayed weak fluorescence throughout the analysis (Fig. 4D and S17 $\dagger$ ). This result confirmed the tumor targeting ability of the tripartite DNA probe due to the FAP moiety. Another control with the non-amplification probe Y-H1-FAP also did not display a substantial fluorescence response, implying the significant role of the HCR circuit in in vivo RNA imaging. Furthermore, we merely obtained slightly increased fluorescence signals after administration of the tripartite DNA probe in the MCF-7 tumor-bearing mice (Fig. 4D and S17†). This result verified the specificity of the tripartite DNA probe in targeting cancer cells with folate receptor positive expression.

To gain insight into the fluorescence response kinetics, we then performed prolonged time-dependent studies of the HeLa tumor bearing mice via tail vein injection of the tripartite DNA probe. It was observed that the maximum response was achieved at $1 \mathrm{~h}$ post-injection and subsequently the fluorescence

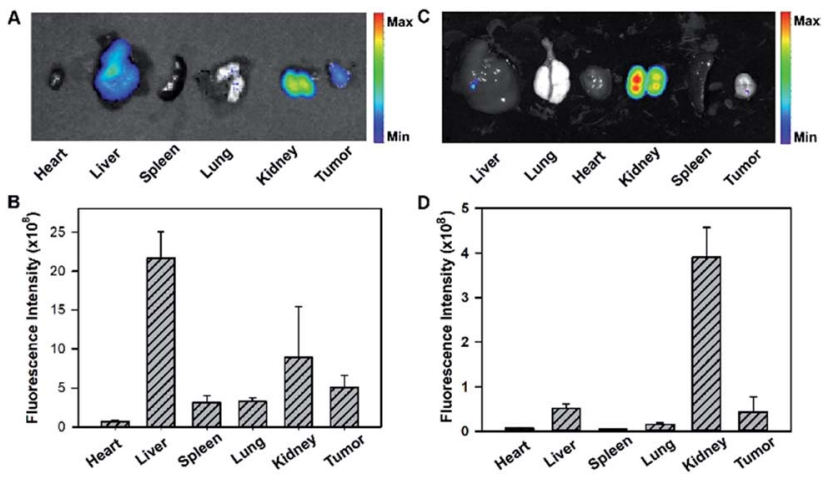

Fig. 5 Fluorescent images and intensity analysis of tumor and main

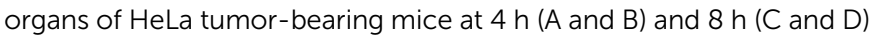
post-injection of the tripartite $\mathrm{Y}-\mathrm{H} 1-\mathrm{H} 2-\mathrm{FAP}$ probe. Error bars were obtained from three repetitive experiments.

decreased until approaching the background level at $8 \mathrm{~h}$ (Fig. S18 $\dagger$ ). This finding suggested the rapid activation of the fluorescence response in the tumor area and fast clearance of the probes and the HCR products. Moreover, ex vivo fluorescence imaging of organs dissected at $4 \mathrm{~h}$ post-injection, such as the heart, liver, spleen, lung, kidney and tumor, revealed that the activated fluorescence signals were dominantly obtained in the liver, kidney and tumor (Fig. 5A and B). This finding indicated that the probes and the HCR products were mainly metabolized and cleared in the liver and kidney. Ex vivo fluorescence imaging of organs dissected at $8 \mathrm{~h}$ post-injection gave different distributions of the probes and the HCR products, and the fluorescence only resided in the kidney at this time (Fig. 5C and D). Presumably, this result can be attributed to the rapid metabolism of the probe in the liver and slow excretion of the HCR product from the tumor into the kidney. Taken together, the live mouse imaging demonstrated that the tripartite DNA probe enabled selective accumulation in tumors with positive folate receptor expression and delivered activated fluorescence signals in response to miR-21 expression in living animals. With the combination of different tumor-targeting ligands, the tripartite DNA probe provides a valuable platform for RNA imaging and related in vivo tumor diagnostics and treatment evaluation.

\section{Conclusions}

In summary, we developed a novel tripartite DNA probe that enabled fluorescent RNA imaging in living mice via an in vivo hybridization chain reaction (HCR) circuit. The Y-shaped tripartite DNA probe allowed facile modular synthesis with a high yield. It also demonstrated high sensitivity and specificity in in vitro detection of target RNA with a subpicomolar detection limit. The tripartite DNA probe was demonstrated to be selectively and efficiently internalized into cells with FA receptor overexpression via a caveolar-mediated endocytosis mechanism, affording the potential for efficient escape from lysosomal degradation. Live cell imaging revealed that the probe displayed fluorescence signals dynamically correlated with miR- 
21 expression. The tripartite DNA probe also showed increased stability in serum and enabled specific delivery into tumor cells for high-contrast imaging of miR-21 in living mice. Because of the broad utility of DNA amplification circuits for RNA detection, the tripartite DNA probe design may open the door for intracellular RNA imaging in living animals using DNAminimal structures. To our knowledge, it is the first time that DNA probes have been developed for in vivo imaging of RNA in living mice. In virtue of the broad utility of DNA amplification circuits for ultrasensitive RNA detection, the tripartite DNA design may open the door for intracellular RNA imaging in living animals, implying its potential for tumor biology studies and theranostics.

\section{Conflicts of interest}

There are no conflicts to declare.

\section{Acknowledgements}

All animal operations were in accord with institutional animal use and care regulations, according to protocol No. SYXK (Xiang) 2008-0001, approved by the Laboratory Animal Center of Hunan. This work was supported by the National Natural Science Foundation of China (Grants 21525522 and 21705039).

\section{Notes and references}

1 B. R. Smith and S. S. Gambhir, Chem. Rev., 2017, 117, 901986.

2 M. Gao, F. Yu, C. Lv, J. Choo and L. Chen, Chem. Soc. Rev., 2017, 46, 2237-2271.

3 J. Zhang, L. P. Smaga, N. S. R. Satyavolu, J. Chan and Y. Lu, J. Am. Chem. Soc., 2017, 139, 17225-17228.

4 D. Asanuma, M. Sakabe, M. Kamiya, K. Yamamoto, J. Hiratake, M. Ogawa, N. Kosaka, P. L. Choyke, T. Nagano, H. Kobayashi and Y. Urano, Nat. Commun., 2015, 6, 6463.

5 C. E. Callmann, C. V. Barback, M. P. Thompson, D. J. Hall, R. F. Mattrey and N. C. Gianneschi, Adv. Mater., 2015, 27, 4611-4615.

6 Y. Feng, et al., Chem. Sci., 2017, 8, 3703-3711.

7 J. A. Varela, J. P. Dupuis, L. Etchepare, A. Espana, L. Cognet and L. Groc, Nat. Commun., 2016, 7, 10947.

8 P. Gao, W. Pan, N. Li and B. Tang, Chem. Sci., 2019, 10, 60356071.

9 A. Zomer, C. Maynard, F. J. Verweij, A. Kamermans, R. Schäfer, E. Beerling, R. M. Schiffelers, E. de Wit, J. Berenguer, S. I. J. Ellenbroek, T. Wurdinger, D. M. Pegtel and J. van Rheenen, Cell, 2015, 161, 1046-1057.

10 X. Zheng, X. Wang, H. Mao, W. Wu, B. Liu and X. Jiang, Nat. Commun., 2015, 6, 5834.

11 R. Kole, A. R. Krainer and S. Altman, Nat. Rev. Drug Discovery, 2012, 11, 125-140.

12 S. Tyagi, Nat. Methods, 2009, 6, 331-338.

13 N. Daigle and J. Ellenberg, Nat. Methods, 2007, 4, 633-636.

14 J. Zheng, R. Yang, M. Shi, C. Wu, X. Fang, Y. Li, J. Li and W. Tan, Chem. Soc. Rev., 2015, 44, 3036-3055.
15 H. Zhang, F. Li, B. Dever, X.-F. Li and X. C. Le, Chem. Rev., 2013, 113, 2812-2841.

16 T. L. Halo, K. M. McMahon, N. L. Angeloni, Y. Xu, W. Wang, A. B. Chinen, D. Malin, E. Strekalova, V. L. Cryns, C. Cheng, C. A. Mirkin and C. S. Thaxton, Proc. Natl. Acad. Sci. U. S. A., 2014, 111, 17104-17109.

17 H. Li, B. Zhang, X. Lu, X. Tan, F. Jia, Y. Xiao, Z. Cheng, Y. Li, D. O. Silva, H. S. Schrekker, K. Zhang and C. A. Mirkin, Proc. Natl. Acad. Sci. U. S. A., 2018, 115, 4340-4344.

18 Z.-M. Ying, Z. Wu, B. Tu, W. Tan and J.-H. Jiang, J. Am. Chem. Soc., 2017, 139, 9779-9782.

19 R. L. Strack, M. D. Disney and S. R. Jaffrey, Nat. Methods, 2013, 10, 1219-1224.

20 T. Lionnet, K. Czaplinski, X. Darzacq, Y. Shav-Tal, A. L. Wells, J. A. Chao, H. Y. Park, V. de Turris, M. LopezJones and R. H. Singer, Nat. Methods, 2011, 8, 165-170.

21 H. Y. Park, H. Lim, Y. J. Yoon, A. Follenzi, C. Nwokafor, M. Lopez-Jones, X. Meng and R. H. Singer, Science, 2014, 343, 422-424.

22 I. Oomoto, A. Suzuki-Hirano, H. Umeshima, Y.-W. Han, H. Yanagisawa, P. Carlton, Y. Harada, M. Kengaku, A. Okamoto, T. Shimogori and D. O. Wang, Nucleic Acids Res., 2015, 43, e126.

23 R. Weissleder, A. Moore, U. Mahmood, R. Bhorade, H. Benveniste, E. A. Chiocca and J. P. Basilion, Nat. Med., 2000, 6, 351-355.

24 Y. Xia, R. Zhang, Z. Wang, J. Tian and X. Chen, Chem. Soc. Rev., 2017, 46, 2824-2843.

25 J. Li, S. Liu, L. Sun, W. Li, S.-Y. Zhang, S. Yang, J. Li and H.-H. Yang, J. Am. Chem. Soc., 2018, 140, 16589-16595.

26 H. M. T. Choi, C. R. Calvert, N. Husain, D. Huss, J. C. Barsi, B. E. Deverman, R. C. Hunter, M. Kato, S. M. Lee, A. C. T. Abelin, A. Z. Rosenthal, O. S. Akbari, Y. Li, B. A. Hay, P. W. Sternberg, P. H. Patterson, E. H. Davidson, S. K. Mazmanian, D. A. Prober, M. van de Rijn, J. R. Leadbetter, D. K. Newman, C. Readhead, M. E. Bronner, B. Wold, R. Lansford, T. Sauka-Spengler, S. E. Fraser and N. A. Pierce, Development, 2016, 143, 36323637.

27 H. M. T. Choi, M. Schwarzkopf, M. E. Fornace, A. Acharya, G. Artavanis, J. Stegmaier, A. Cunha and N. A. Pierce, Development, 2018, 145, dev165753.

28 Z. Wu, G.-Q. Liu, X.-L. Yang and J.-H. Jiang, J. Am. Chem. Soc., 2015, 137, 6829-6836.

29 H. Elnakat and M. Ratnam, Adv. Drug Delivery Rev., 2004, 56, 1067-1084.

30 M. B. Baker, G. Bao and C. D. Searles, Nucleic Acids Res., 2012, 40, e13.

31 Y. Li, Y. D. Tseng, S. Y. Kwon, L. d'Espaux, J. S. Bunch, P. L. McEuen and D. Luo, Nat. Mater., 2004, 3, 38-42.

32 S. Yue, X. Song, W. Song and S. Bi, Chem. Sci., 2019, 10, 16511658.

33 K. Kacprzak, I. Skiera, M. Piasecka and Z. Paryzek, Chem. Rev., 2016, 116, 5689-5743.

34 J. A. Chan, A. M. Krichevsky and K. S. Kosik, Cancer Res., 2005, 65, 6029-6033. 
35 R. Zhao, B. Wang, X. Yang, Y. Xiao, X. Wang, C. Shao and R. Tang, Angew. Chem., Int. Ed., 2016, 55, 5225-5229.

36 E. Leone, E. Morelli, M. T. Di Martino, N. Amodio, U. Foresta, A. Gullà, M. Rossi, A. Neri, A. Giordano, N. C. Munshi, K. C. Anderson, P. Tagliaferri and P. Tassone, Clin. Cancer Res., 2013, 19, 2096-2106.

37 H. Dong, J. Lei, H. Ju, F. Zhi, H. Wang, W. Guo, Z. Zhu and F. Yan, Angew. Chem., Int. Ed., 2012, 51, 4607-4612.

38 J. Lu, G. Getz, E. A. Miska, E. Alvarez-Saavedra, J. Lamb, D. Peck, A. Sweet-Cordero, B. L. Ebert, R. H. Mak, A. A. Ferrando, J. R. Downing, T. Jacks, H. R. Horvitz and T. R. Golub, Nature, 2005, 435, 834-838.
39 D. Feng, Y. Song, W. Shi, X. Li and H. Ma, Anal. Chem., 2013, 85, 6530-6535.

40 F. Zhao, Y. Zhao, Y. Liu, X. Chang, C. Chen and Y. Zhao, Small, 2011, 7, 1322-1337.

41 C. Dalal, A. Saha and N. R. Jana, J. Phys. Chem. C, 2016, 120, 6778-6786.

42 J. W. Conway, C. K. McLaughlin, K. J. Castor and H. Sleiman, Chem. Commun., 2013, 49, 1172-1174.

43 A. Lacroix, E. Vengut-Climent, D. de Rochambeau and H. F. Sleiman, ACS Cent. Sci., 2016, 5, 882-891. 